\title{
An algebraic approach for solving boundary value màtrix problems: existence, uniqueness and closed form solutions
}

\author{
LUCAS JÓDAR
}

\begin{abstract}
In this paper we show that in an analogous way to the scalar case, the general solution of a non homogeneous second order matrix differential equation may be expressed in terms of the exponential functions of certain matrices related to the corresponding characteristic algebraic matrix equation. We introduce the concept of co-solution of an algebraic equation of the type $X^{2}+A_{1} X+A_{0}=0$, that allows us to obtain a method of the variation of the parameters for the matrix case and further to find existence, uniqueness conditions for solutions of boundary value problems. These conditions are of algebraic type, involving the Penrose-Moore pseudoinverse of a matrix related to the problem. A computable closed form for solutions of the problem is given.
\end{abstract}

\section{INTRODUCTION}

Second order matrix differential equations with constant coefficients appear in the study of vibrational systems $[6,11]$, electrical, mechanical and thermal problems [14], as well as when one considers finite approximations to distributed parameter systems described by partial differential equations [2].

It is well known that the solution of the Cauchy problem

$$
X^{(2)}(t)+A_{1} X^{(1)}(t)+A_{0} X(t)=F(t) X(0)=C_{0}, X^{(1)}(0)=C_{1}
$$

where $A_{i}, C_{i}, i=1,2, F(t)$ and $X(t)$ are $n \times n$ complex matrices, elements of

1980 Mathematics Subject Classification (1985 revision): 15A24, 34B05, 34B10, $15 \mathrm{~A} 09$.

Editorial de la Universidad Complutense. Madrid, 1988. 
$\mathbb{C}_{n \times n}$ may be solved considering the standard change $X=Y_{1}, X^{(1)}=Y_{2}$ and the equivalent first order extended system

$$
Y=\left[\begin{array}{l}
Y_{1} \\
Y_{2}
\end{array}\right] ; \quad C_{L}=\left[\begin{array}{cc}
0 & I \\
-A_{0} & -A_{1}
\end{array}\right] ; \quad Y^{(1)}(t)=C_{L} Y(t)+\left[\begin{array}{c}
0 \\
F(t)
\end{array}\right] ; \quad Y(0)=\left[\begin{array}{c}
C_{0} \\
C_{1}
\end{array}\right]
$$

If $F(t)$ is continuous, then the unique solution of problem (1.1) is given by the expression

$$
X(t)=[I, 0] \exp \left(t C_{L}\right)\left\{\left[\begin{array}{l}
C_{0} \\
C_{1}
\end{array}\right]+\int_{0}^{t} \exp \left(-s C_{L}\right)\left[\begin{array}{c}
0 \\
F(s)
\end{array}\right] d s\right\}
$$

see $[5$, p. 122], for instance. The expression (1.3) for the solution of problem (1.1) has some numerical and theoretical inconvenients. So, the expression (1.3) involves the increase of the dimension of the problem. Also, the expression (1.3) is not totally explicit because of the existence of the pre-factor $[1,0]$, and the exponential $\exp \left(t C_{L}\right)$ is not known in terms of data.

These inconvenients make that expression (1.3) is not useful for the study of boundary value problems related to the matrix differential equation

$$
X^{(2)}(t)+A_{1} X^{(1)}(t)+A_{0} X(t)=F(t)
$$

This motivates a different approach to the boundary value problem. In recent papers $[7,9,10]$, and in an analogous way to the scalar case, explicit solutions of Cauchy problems and boundary value problems related to equation (1.4) are given in terms of a pair of solutions $X_{0}, X_{1}$, of the matrix equation

$$
X^{2}+A_{1} X+A_{0}=0
$$

such that the difference $X_{1}-X_{0}$ is invertible. However, the method developed in $[7,9,10]$, as well as the one of $[8]$, has the inconvenient that equation (1.5) may be unsolvable, or that a pair of solutions $X_{0}, X_{1}$, with $X_{1}-X_{0}$ invertible, is not available. For instance, if $A_{1}=0$ and $A_{0}$ has not square roots, [4], then the corresponding equation (1.5) is unsolvable.

In order to study boundary value problems related to equation (1.5), when the algebraic equation (1.5) is possibly unsolvable, we introduce the concept of co-solution of the algebraic equation (1.5). This generalizes the concept of solution of the algebraic equation (1.5) and it allows us to represent the general solution of the homogeneous equation

$$
X^{(2)}(t)+A_{1} X^{(1)}(t)+A_{0} X(t)=0
$$


in terms of an appropriate pair of co-solutions of equation (1.5). In section 3 a generalized variation of the parameters method for solving equation (1.4) is given. We apply this method in order to find existence and uniqueness conditions_for_solutions of the boundary value problem

$$
\left.\begin{array}{r}
X^{(2)}(t)+A_{1} X^{(1)}(t)+A_{0} X(t)=F(t) \\
E_{1} X(0)+E_{2} X^{(1)}(0)=G_{1} \\
F_{1} X(a)+F_{2} X^{(1)}(a)=G_{2} \\
0 \leqslant t \leqslant a
\end{array}\right\}
$$

where $E_{i}, F_{i}, G_{i}$, for $i=1,2$, and $A_{j}$, for $j=0,1, F(t), X(t)$, are matrices in $\mathbb{C}_{n \times n}$. By using an appropriate pair of co-solutions of equation (1.5), the problem $(1.7)$ is transformed into an algebraic system, then considering generalized inverses of matrices, a representation for the general solution of the boundary value problem (1.7) is obtained.

If $A$ is a matrix in $\mathbb{C}_{n \times m}$, we represent by $A^{+}$the Penrose-Moore pseudoinverse of $A$. An account of the uses and properties of this concept may be found in [13].

\section{ON THE GENERAL SOLUTION \\ OF THE MATRIX EQUATION $X^{(2)}(t)+A_{1} X^{(1)}(t)+A_{0} X(t)=0$}

We begin this section by introducing the concept of co-solution of the equation (1.5).

Definition 1.1. Let us consider the equation (1.5) where $A_{i} \in \mathbb{C}_{n \times n}$, for $i=0,1$. We say that a pair $(X, T)$ of matrices in $\mathbb{C}_{n \times n}$ is a co-solution of equation (1.5) if $X \neq 0$ and satisfies

$$
X T^{2}+A_{1} X T+A_{0} X=0
$$

Example 1. Let us suppose that $T \in \mathbb{C}_{n \times n}$ is a solution of equation (1.5), if $I$ is the identity matrix in $\mathbb{C}_{n \times n}$, then the pair $(I, I)$ is a co-solution of equation (1.5).

Example 2. Let $z$ be an eigenvalue of the companion matrix $C_{L}$ defined in (1,2). From [6], p. 14, the matrix $z^{2} I+A_{1} z+A_{0}$ is singular. Thus there exists non zero matrices $X$ such that $\left(z^{2} I+A_{1} z+A_{0}\right) X=0$. So, the pair $(X, z I)$ is a co-solution of equation (1.5). 
Definition 1.2. Let $\left(X_{i}, T_{i}\right)$ be co-solutions of equation (1:5), for $i=1,2$. We say that $\left(X_{i}, T_{i}\right), i=1,2$, is a fundamental pair of co-solutions of equation (1.5), if the block matrix $V$ defined by

$$
V=\left[\begin{array}{cc}
X_{1} & X_{2} \\
X_{1} T_{1} & X_{2} T_{2}
\end{array}\right]
$$

is invertible in $\mathbb{C}_{2 n \times 2 n}$.

Example 3. Let us suppose that $T_{1}$, and $T_{2}$ are two solutions of equation (1.5), then the pair $\left\{\left(I, T_{1}\right),\left(I, T_{2}\right)\right\}$ define a fundamental pair of co-solutions of equation (1.5), if and only if, the matrix $T_{2}-T_{1}$ is invertible, see lemma 1 of $[8]$.

Next theorem shows that for a very general class of equation of the type (1.5) a fundamental pair of co-solutions is available and provides a method for obtaining fundamental pairs of co-solutions.

Theorem 1. Let $A_{0} A_{1}$ be matrices in $\mathbb{C}_{n \times n}$ and let $C_{L}$ the companion matrix defined by (1.2). If the matrix $C_{L}$ is similar to a block diagonal matrix $J=\left[\begin{array}{ll}J_{1} & 0 \\ 0 & J_{2}\end{array}\right]$, where $J_{i}$ for $i=1,2$, are matrices in $\mathbb{C}_{n \times n}$, then equation (1.5) has a fundamental pair of co-solutions. If $P=\left(P_{i j}\right)$, with $P_{i j} \in \mathbb{C}_{n \times n}$, for $1 \leqslant i, j \leqslant 2$, is an invertible matrix in $\mathbb{C}_{2 n \times 2 n}$ such that $P J=C_{L} P$, then $\left(P_{11}, J_{1}\right),\left(P_{12}, J_{2}\right)$, is a fundamental pair of co-solutions of equation (1.5).

Proof. Let $P=\left(P_{i j}\right)$, with $P_{i j} \in \mathbb{C}_{n \times n}, 1 \leqslant i, j \leqslant 2$, an invertible block partitioned matrix satisfying $P J=C_{L} P$. From the equality

$$
\left[\begin{array}{ll}
P_{11} & P_{12} \\
P_{21} & P_{22}
\end{array}\right]\left[\begin{array}{ll}
J_{1} & 0 \\
0 & J_{2}
\end{array}\right]=\left[\begin{array}{cc}
0 & I \\
-A_{0} & -A_{1}
\end{array}\right]\left[\begin{array}{ll}
P_{11} & P_{12} \\
P_{21} & P_{22}
\end{array}\right]
$$

we have

$$
\begin{gathered}
P_{11} J_{1}=P_{21} \\
P_{21} J_{1}=-A_{0} P_{11}-A_{1} P_{21} \\
P_{12} J_{2}=P_{22} \\
P_{22} J_{2}=-A_{0} P_{12}-A_{1} P_{22}
\end{gathered}
$$

From (2.3) and (2.4) we have $P_{11} J_{1}^{2}=-A_{0} P_{11}-A_{1} P_{11} J_{1}$, and from (2.5). (2.6) one gets $P_{12} J_{2}^{2}=-A_{0} P_{12}-A_{1} P_{12} J_{2}$. Thus, $\left\{\left(P_{11}, J_{1}\right),\left(P_{12}, J_{2}\right)\right\}$ is a fundamental pair of co-solutions of equation (1.5), because from $(2.3)-(2.6)$ and 
the invertibility of $P$, it follows that $P_{11} \neq 0$ and $P_{12} \neq 0$. Note also that the matrix $V$ defined by $(2.2)$ is invertible because

$$
V=\left[\begin{array}{cc}
P_{11} & P_{12} \\
P_{11} J_{1} & P_{12} J_{2}
\end{array}\right]=\left[\begin{array}{cc}
P_{11} & P_{12} \\
P_{21} & P_{22}
\end{array}\right]=P
$$

Thus the result is concluded.

Remark 1. From theorem 1, in order to know the existence of a fundamental pair of co-solutions of equation (1.5), only it is required that the Jordan matrix of the companion matrix $C_{L}$ may be expressed as a block diagonal matrix with two blocks in the diagonal of dimension $n$. This information is available from the characteristic polynomial of $C_{L}$. If the condition of theorem 1 is satisfied, in order to construct a fundamental pair of co-solutions, we need to compute the matrices $P_{11}$ and $P_{12}$, but it is an easy matter because the columns of $P$ are the vectors of a Jordan basis of the matrix $C_{L},[12]$, chapter 6.

Theorem 2. Let us suppose that equation (1.5) has a fundamental pair $\left(X_{i}, T_{i}\right)$ $i=1,2$, of co-solutions. Then the unique solution of problem (1.1) takes the form

$$
X(t)=X_{1} \exp \left(t T_{1}\right) D_{1}+X_{2} \exp \left(t T_{2}\right) D_{2}
$$

where

$$
\left[\begin{array}{l}
D_{1} \\
D_{2}
\end{array}\right]=V^{-1}\left[\begin{array}{l}
C_{0} \\
C_{1}
\end{array}\right]
$$

and $V$ is given by (2.2).

Proof. If we denote $Y_{i}(t)=X_{i} \exp \left(t T_{i}\right) D_{i}$, for $i=1,2$, and arbitrary matrices $D_{i} \in \mathbb{C}_{n \times n}$ for $i=1,2$, it follows that

$$
Y_{i}^{(1)}(t)=X_{i} T_{i} \exp \left(t T_{i}\right) D_{i}, Y_{i}^{(2)}(t)=X_{i} T_{i}^{2} \exp \left(t T_{i}\right) D_{i}, i=1,2
$$

Hence we have

$$
Y_{i}^{(2)}(t)+A_{1} Y_{i}^{(1)}(t)+A_{0} Y_{i}(t)=\left(X_{i} T_{i}^{2}+A_{1} X_{i} T_{i}+A_{0} X_{i}\right) \exp \left(t T_{i}\right) D_{i}=0
$$

because $\left(X_{i} T_{i}\right)$ is a co-solution of $(1.5)$, for $i=1,2$. Thus for any matrices $D_{1}, D_{2}$ in $\mathbb{C}_{n \times n}$, the matrix function $X(t)$ defined by $(2.7)$ is a solution of the differential equation (1.6). In order to satisfy the Cauchy conditions of (1.1), the matrices $D_{1}, D_{2}$, must verify the system 


$$
\begin{aligned}
X(0) & =C_{0}=X_{1} D_{1}+X_{2} D_{2} \\
X^{(1)}(0) & =C_{1}=X_{1} T_{1} D_{1}+X_{2} T_{2} D_{2}
\end{aligned}
$$

or equivalently

$$
\left[\begin{array}{l}
C_{0} \\
C_{1}
\end{array}\right]=\left[\begin{array}{cc}
X_{1} & X_{2} \\
X_{1} T_{1} & X_{2} T_{2}
\end{array}\right]\left[\begin{array}{l}
D_{1} \\
D_{2}
\end{array}\right]
$$

From the uniqueness for solutions of the Cauchy problem (1.1) and from (2.9) the result is established.

\section{EXISTENCE, UNIQUENESS AND EXPLICIT SOLUTIONS OF BOUNDARY VALUE PROBLEMS}

Theorem 2 suggests that in analogous way to the scalar case we can obtain a method of variation of parameters in order to find the general solution of equation (1.4). Let us consider equation (1.4), where $F(t)$ defines a continuous matrix function with values in $\mathbb{C}_{n \times n}$, on an interval containing the origin. Let us suppose that in an analogous way to the scalar case, we are interested in finding appropriate matrix functions $D_{i}(t)$, for $i=1,2$, such that the function

$$
X(t)=\sum_{i=1}^{2} X_{i} \exp \left(t T_{i}\right) D_{i}(t)
$$

is a solution of equation (1.4). Let us assume that we choose the functions $D_{i}(t)$ such that

$$
\left[\begin{array}{cc}
X_{1} \exp \left(t T_{1}\right) & X_{2} \exp \left(t T_{2}\right) \\
X_{1} T_{1} \exp \left(t T_{1}\right) & X_{2} T_{2} \exp \left(t T_{2}\right)
\end{array}\right]\left[\begin{array}{l}
D_{1}^{(1)}(t) \\
D_{2}^{(1)}(t)
\end{array}\right]=\left[\begin{array}{l}
0 \\
F(t)
\end{array}\right]
$$

that may be written as

$$
\left[\begin{array}{cc}
X_{1} & X_{2} \\
X_{1} T_{1} & X_{2} T_{2}
\end{array}\right]\left[\begin{array}{cc}
\exp \left(t T_{1}\right) & 0 \\
0 & \exp \left(t T_{2}\right)
\end{array}\right]\left[\begin{array}{c}
D_{1}^{(t)}(t) \\
D_{2}^{(1)}(t)
\end{array}\right]=\left[\begin{array}{c}
0 \\
F(t)
\end{array}\right]
$$

If we assume that $\left(X_{1} T_{1}\right),\left(X_{2}, T_{2}\right)$ is a fundamental pair of co-solutions of equation (1.5), then the matrix $V$ defined by (2.2) is invertible in $\mathbb{C}_{2 n \times 2 n}$, and if we denote by $W=\left\langle W_{i j}\right)$, for $1 \leqslant i, j \leqslant 2$, with $W_{i j} \in \mathbb{C}_{n \times n}$, the inverse matrix of $V$, 
with the same dimensional block partition as $V$, then from (3.3) it follows that

$$
\left[\begin{array}{l}
D_{1}(t) \\
D_{2}(t)
\end{array}\right]=\left[\begin{array}{c}
D_{2}(0) \\
D_{2}(0)
\end{array}\right]+\int_{0}^{t}\left[\operatorname{Diag}\left(\exp \left(-s T_{1}\right), \exp \left(-s T_{2}\right)\right)\right] W\left[\begin{array}{c}
0 \\
F(s)
\end{array}\right] d s
$$

Note that

$$
\left[\mathrm{Diag}\left(\exp \left(-s T_{1}\right), \exp \left(-s T_{2}\right)\right] W\left[\begin{array}{c}
0 \\
F(s)
\end{array}\right]=\left[\begin{array}{l}
\exp \left(-s T_{1}\right) W_{12} F(s) \\
\exp \left(-s T_{2}\right) W_{22} F(s)
\end{array}\right]\right.
$$

Hence and from (3.4) one gets

$$
\begin{aligned}
& D_{1}(t)=D_{1}(0)+\int_{0}^{t} \exp \left(-s T_{1}\right) W_{12} F(s) d s \\
& D_{2}(t)=D_{2}(0)+\int_{0}^{t} \exp \left(-s T_{2}\right) W_{22} F(s) d s
\end{aligned}
$$

Note that from (3.2), the derivatives $X^{(i)}(t)$ of $X(t)$ defined by (3.1) takes the expressions

and

$$
\begin{gathered}
X^{(1)}(t)=X_{1} T_{1} \exp \left(t T_{1}\right) D_{1}(t)+X_{2} T_{2} \exp \left(t T_{2}\right) D_{2}(t) \\
X^{(2)}(t)=X_{1} T_{1}^{2} \exp \left(t T_{1}\right) D_{1}(t)+X_{2} T_{2}^{2} \exp \left(t T_{2}\right) D_{2}(t)+F(t)
\end{gathered}
$$

$$
\begin{gathered}
X^{(2)}(t)+A_{1} X^{(1)}(t)+A_{0} X(t)= \\
=\left(X_{1} T_{1}^{2}+A_{1} X_{1} T_{1}+A_{0} X_{1}\right) \exp \left(t T_{1}\right) D_{1}(t)+\left(X_{2} T_{2}^{2}+A_{1} X_{2} T_{2}+A_{0} X_{2}\right) \\
\exp \left(t T_{2}\right) D_{2}(t)+F(t)=F(t)
\end{gathered}
$$

because of (3.6) and $X_{i} T_{i}^{2}+A_{1} X_{i} T_{i}+A_{0} X_{i}=0$, for $i=1,2$. Thus, if $D_{i}(t)$, for $i$ $=1,2$, are defined by $(3.5)$, where $D_{i}(0), i=1,2$, are arbitrary matrices in $\mathbb{C}_{n \times n}$, the matrix function $X(t)$ defined by (3.1) is a solution of equation (1.4).

If $Y(t)$ is a solution of equation (1.4) such that $Y(0)=C_{0}, \bar{Y}^{(1)}(0)=C_{1}$. Then we impose to the functions $D_{i}(t), i=1,2$, that $X(t)$ defined by (3.1) satisfies the same initial conditions that $Y(t)$, this is, taking $t=0$ in $(3.1),(3.6)$, one gets

$$
C_{0}=X_{1} D_{1}(0)+X_{2} D_{2}(0) \text { and } C_{1}=X_{1} T_{1} D_{1}(0)+X_{2} T_{2} D_{2}(0)
$$

Hence we have that $D_{i}(0), i=1,2$, must be given by

$$
\left[\begin{array}{l}
D_{1}(0) \\
D_{2}(0)
\end{array}\right]=V^{-1}\left[\begin{array}{l}
C_{0} \\
C_{1}
\end{array}\right]
$$


As $X(t)$ defined by (3.1) with $D_{i}(0)$, for $i=1,2$, defined by (3.8), satisfies the same initial conditions that $Y(t)$, from the uniqueness property, $Y(t)$ and $X(t)$ coincides. This proves that the expression (3.1), (3.5), represents the general solution of equation (1.4). The following result has been proved:

Theorem 3. Let $F(t)$ be a continuous $\mathbb{C}_{n \times n}$ valued function defined on a interval $U$ of the real line containing the origin. Let us assume that equation (1.5) has a fundamental pair $\left\{\left(X_{1}, T_{1}\right),\left(X_{2}, T_{2}\right)\right\}$, of co-solutions, and let $V^{-1}=W$ $=\left(W_{i j}\right)$, where $W_{i} \in \mathbb{C}_{n \times n}$, for $1 \leqslant i, j \leqslant 2$, and $V$ is the matrix defined by (2.2). Then the general solution of equation (1.4) is given by the function $X(t)$ defined by (3.1), (3.5), where $D_{i}(0), i=1,2$, are arbitrary matrices in $\mathbb{C}_{n \times n}$.

Now we will show that the representation (3.1), (3.5), for the general solution of equation (1.4), may be used to find existence and uniqueness conditions for solutions of the boundary value problem (1.7), as well as, for obtaining explicit solutions of them in terms of a fundamental pair of cosolutions of equation (1.5).

Theorem 4. Let $F(t)$ be a continuous $\mathbb{C}_{n \times n}$ valued function defined on the interval $[0, a]$, with $a>0$, and let us suppose that equation (1.5) has a fundamental pair of co-solutions $\left\{\left(X_{1}, T_{1}\right),\left(X_{2}, T_{2}\right)\right\}$. Let $W=\left(W_{i j}\right), W_{i j} \in \mathbb{C}_{n \times n}$, be the inverse of the matrix $V$ defined by (2,2), and let $Q$ be the matrix

$$
\begin{aligned}
Q=G_{2}-\left(F_{1} X_{1}+F_{2} X_{1} T_{1}\right) \int_{0}^{a} \exp \left((a-s) T_{1}\right) W_{12} F(s) d s- \\
\left(F_{1} X_{2}+F_{2} X_{2} T_{2}\right) \int_{0}^{a} \exp \left((a-s) T_{2}\right) W_{22} F(s) d s
\end{aligned}
$$

where $G_{2}$ is the matrix appearing in (1.7). Then the boundary value problem (1.6) is solvable, if and only if, the matrix $S$ defined by

$$
S=\left[\begin{array}{cc}
E_{1} X_{1}+E_{2} X_{1} T_{1} & E_{1} X_{2}+E_{2} X_{2} T_{2} \\
\left(F_{1} X_{1}+F_{2} X_{\mathrm{t}} T_{1}\right) \exp \left(a T_{1}\right) & \left(F_{1} X_{2}+F_{2} X_{2} T_{2}\right) \exp \left(a T_{2}\right)
\end{array}\right]
$$

satisfies the property

$$
S S^{+}\left[\begin{array}{l}
G_{1} \\
Q
\end{array}\right]=\left[\begin{array}{l}
G_{1} \\
Q
\end{array}\right]
$$

Also, if the condition (3.11) is satisfied, then the solution set of problem (1.7) is given by the functions $X(t)$ defined by $(3.1),(3.5)$, where $D_{1}(0), D_{2}(0)$ take the form 


$$
\left[\begin{array}{c}
D_{1}(0) \\
D_{2}(0)
\end{array}\right]=S^{+}\left[\begin{array}{c}
G_{1} \\
Q
\end{array}\right]+\left(I_{2 n}-S^{+} S\right) Y
$$

and $Y$ is an arbitrary matrix in $\mathbb{C}_{2 n \times n}$.

Proof. From theorem 3, the general solution of equation (1.4) is given by the function $X(t)$ defined by (3.1), (3.5), where $D_{1}(0)$ and $D_{2}(0)$ are arbitrary matrices in $\mathbb{C}_{n \times n}$. In order to find solutions of problem (1.7), we have to find appropriate matrices $D_{1}(0), D_{2}(0)$ in $\mathbb{C}_{n \times n}$, such that the corresponding function $X(t)$, satisfies the boundary value conditions of (1.7). Taking into account that the functions $D_{i}(t), i=1,2$, defined by (3.5), satisfy

and

$$
D_{1}(a)=D_{1}(0)+\int_{0}^{a} \exp \left(-s T_{1}\right) W_{12} F(s) d s
$$

$$
D_{2}(a)=D_{2}(0)+\int_{0}^{a} \exp \left(-s T_{2}\right) W_{22} F(s) d s
$$

by impossing the boundary value conditions of (1.6) to the expression of $X(t)$, it follows that $D_{1}(0)$ and $D_{2}(0)$ must verify

$$
\begin{gathered}
E_{1}\left(X_{1} D_{1}(0)+X_{2} D_{2}(0)\right)+E_{2}\left(X_{1} T_{1} D_{1}(0)+X_{2} T_{2} D_{2}(0)\right)=G_{1} \\
F_{1}\left(X_{1} \exp \left(a T_{1}\right) D_{1}(0)+X_{2} \exp \left(a T_{2}\right) D_{2}(0)\right)+ \\
+F_{2}\left(X_{1} T_{1} \exp \left(a T_{1}\right) D_{1}(0)+X_{2} T_{2} \exp \left(a T_{2}\right) D_{2}(0)\right)=Q
\end{gathered}
$$

where $Q$ is defined by (3.9). Thus, $D_{i}(0)$, for $i=1,2$, must verify the algebraic system

$$
S\left[\begin{array}{l}
D_{1}(0) \\
D_{2}(0)
\end{array}\right]=\left[\begin{array}{l}
G_{1} \\
Q
\end{array}\right]
$$

It is well known, [13], p. 24, that system (3.14) is solvable, if and only if, the condition (3.11) is satisfied, and that in this case the solution set of system (3.14) is given by (3.12). Hence the result is established.

Remark 2. In order to find existence conditions for the problem (1.7), we have to check if the condition (3.11) is satisfied. Thus, we have to compute $S^{+}$, an easy method for computing the Penrose-Moore pseudoinverse of a matrix may be found in [3], p. 12.

If the matrix $S$ defined by (3.10) is invertible in $\mathbb{C}_{2 n \times 2 n}$, then $S^{+} S=I$, and problem $(1.7)$ has only one solution given by $(3.1),(3.5)$, where

$$
\left[\begin{array}{l}
D_{1}(0) \\
D_{2}(0)
\end{array}\right]=S^{-1}\left[\begin{array}{l}
G_{1} \\
Q
\end{array}\right]
$$


Next example illustrates the developed theory and shows that our approach is strictly more general than the one developed in the sequence [7$10]$.

Example 3. Let us consider a problem of the type (1.7) for the case where $A_{1}=0$, and $A_{0}=-\left[\begin{array}{ll}1 & 1 \\ 1 & 1\end{array}\right]$. An easy computation yields that $\sigma\left(A_{0}\right)=\{0,-2\}$ and $\sigma\left(C_{L}\right)=\left\{0,2^{\frac{1}{2}},-2^{\frac{1}{2}}\right\}$, and the minimal polynomial $q(z)$ of $C_{L}$, coincides with its characteristic polynomial $p(z)=z\left(z^{2}-2\right)$. Note that as $\sigma\left(-A_{0}\right)=\{0$, $2\}$, then for any square root $B$ of $-A_{0}$, it follows that $\sigma(B)=\left\{0,2^{\frac{1}{2}}\right\}$, or $\sigma(B)$ $=\left\{0,-2^{\frac{1}{2}}\right\}$. In the first case, the characteristic polynomial of $B$ is $p(z)$ $=\dot{z}\left(z-2^{\frac{1}{2}}\right)$ and then $p(B)=B\left(B-2^{\frac{1}{2}} I\right)=B^{2}-2^{\frac{1}{2}} B=-A_{0}-2^{\frac{1}{2}} B=0$, this is $B$ $=-2^{-\frac{1}{2}} A_{0}$. If $\sigma(B)=\left\{0,-2^{\frac{1}{2}}\right\}$, then its characteristic polynomial is $q(z)=z(z$ $\left.+2^{\frac{1}{2}}\right)$ and $q(B)=B\left(B+2^{\frac{1}{2}}\right)=B^{2}+2^{\frac{1}{2}} B=-A_{0}+2^{\frac{1}{2}} B=0$. So, in this case $B$ $=2^{-\frac{1}{2}} A_{0}$. An easy computation yields that $\pm 2^{-\frac{1}{2}} A_{0}$ are the unique square roots of $-A_{0}$. In consequence $B_{1}=2^{-\frac{1}{2}} A_{0}$ and $B_{0}=-2^{-\frac{1}{2}} A_{0}$ are the unique square roots of $-A_{0}$, and $B_{1}-B_{0}=-2^{\frac{1}{2}} A_{0}$ is singular.

Thus in this case the corresponding equation (1.5) has not a pair of solutions whose difference is invertible. On the other hand, as the characteristic and the minimal polynomial of $C_{L}$ coincide, it follows that the Jordan canonical form of $C_{L}$ is given by the matrix

$$
J=\left[\begin{array}{ll}
J_{1} & 0 \\
0 & J_{2}
\end{array}\right]
$$

where

$$
J_{1}=\left[\begin{array}{ll}
0 & 1 \\
0 & 0
\end{array}\right], \quad J_{2}=\left[\begin{array}{ll}
2^{\frac{1}{2}} & 0 \\
0 & 2^{-\frac{1}{2}}
\end{array}\right]
$$

- An easy computation yields that $C_{L}=P J P^{-1}$, where

$$
P=\left[\begin{array}{rrcc}
1 & 1 & 1 & -1 \\
-1 & -1 & 1 & -1 \\
0 & 1 & 2^{\frac{1}{2}} & 2^{\frac{1}{2}} \\
0 & -1 & 2^{\frac{1}{2}} & 2^{\frac{1}{2}}
\end{array}\right]
$$


Thus, taking $P_{11}=\left[\begin{array}{rr}1 & 1 \\ -1 & -1\end{array}\right], P_{12}=\left[\begin{array}{ll}1 & -1 \\ 1 & -1\end{array}\right]$, it follows that $\left(P_{11}, J_{1}\right)$ and $\left(P_{12}, J_{2}\right)$ define a fundamental pair of co-solutions of equation $X^{2}+A_{0}$ $=0$. Taking concrete values of data in (1.7) one gets a family of examples that can not be studied with the developed technique of [7-10] and for which theorem 4 is applicable.

Acknowledgement. This paper has been partially supported by a grant from the D.G.I.C.Y.T., project PS87-0064.

\section{References}

[1] T. M. APóstol, Explicit Formulas for Solutions of the Second Order Matrix Differential Equation $Y^{(2)}=A Y$, Amer. Math. Monthly, 82, No. 2 (1975), 159-162.

[2] M. J. BaLAS, Trends in Large Space Structure Theory: Fondest Hopes, Wildest Dreams, IEEE Trans. Aut. Control, AC-27 (1982), 522-535.

[3] S. L. CAMPBELL and C. D. MeYER, Jr., Generalized Inverses of Linear Transformations, Pitman Pubs. Co., San Francisco, 1979.

[4] G. W. Cross and P. LANCASTER, Square Roots of Complex Matrices, Linear and Multilinear Algebra, 1 (1974), 280-293.

[5] T. M. FletT, Differential Analysis, Cambridge Univ. Press, 1980.

[6] J. C. GohberG, P. Lancaster and L. Rodman, Matrix Polynomials, Academic Press, 1982.

[7] L. JÓDAR, Boundary Value Problems for Second Order Differential Operator Equations, Linear Algebra and its Appl., 83 (1986), 29-38.

[8] L. JODAR, Explicit Expressions for Sturm-Liouville Operator Problems, Proc. of the Edinburgh Math. Soc., 30 (1987), 301-309.

[9] L. JóDAR, Existence, Uniqueness and Closed Form Solutions for Boundary Value Matrix Problems, Applied Math. Letters, 1 (1988), 147-150.

[10] L. JóDAR, Explicit Solutions for Second Order Operator Differential Equations with Two Boundary Value Conditions, Linear Algebra and its Appl., 103 (1988), 73-86.

[11] P. Lancaster, Lambda Matrices and Vibrating Systems, Pergamon, Oxford, 1966.

[12] P. LANCASTER and M. TISMENETSKY, The Theory of Matrices, Sec. Ed. Academic Press, 1985.

[13] C. R. RAO and S. K. MrTRA, Generalized inverses of Matrices and its Applications, John-Wiley, New York, 1971.

[14] R. C. DorF, Modern Control Systems, Second ed., Addison-Wesley, Reading, MA, 1974.

Department of Applied Mathematics Polytechnical University of Valencia P.O. 22.012, Valencia

Spain
Recibido: 18 de mayo de 1988

Revisado: 13 de septiembre de 1988 